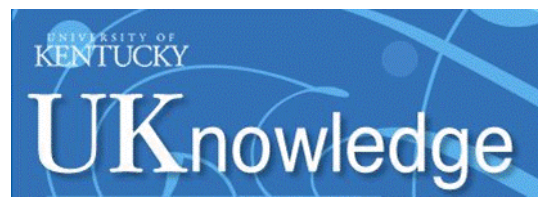

University of Kentucky

UKnowledge

Molecular and Cellular Biochemistry Faculty

Publications

Molecular and Cellular Biochemistry

5-13-2021

\title{
A Screen of FDA-Approved Drugs Identifies Inhibitors of Protein Tyrosine Phosphatase 4A3 (PTP4A3 or PRL-3)
}

\author{
Dylan R. Rivas \\ University of Kentucky, Dylan.Rivas@uky.edu \\ Mark Vincent C. Dela Cerna \\ University of Louisville \\ Caroline N. Smith \\ University of Kentucky, caroline.smith2@uky.edu \\ Shilpa Sampathi \\ University of Kentucky, shilpa.sampathi@uky.edu \\ Blaine G. Patty \\ University of Kentucky, Blaine.Patty@uky.edu
}

Eellowexthis and additionalwworksat:https://uknowledge.uky.edu/biochem_facpub

Part of the Biochemistry, Biophysics, and Structural Biology Commons, Medical Genetics Commons, and the Oncology Commons

Right click to open a feedback form in a new tab to let us know how this document benefits you.

\section{Repository Citation}

Rivas, Dylan R.; Dela Cerna, Mark Vincent C.; Smith, Caroline N.; Sampathi, Shilpa; Patty, Blaine G.; Lee, Donghan; and Blackburn, Jessica S., "A Screen of FDA-Approved Drugs Identifies Inhibitors of Protein Tyrosine Phosphatase 4A3 (PTP4A3 or PRL-3)" (2021). Molecular and Cellular Biochemistry Faculty Publications. 185.

https://uknowledge.uky.edu/biochem_facpub/185

This Article is brought to you for free and open access by the Molecular and Cellular Biochemistry at UKnowledge. It has been accepted for inclusion in Molecular and Cellular Biochemistry Faculty Publications by an authorized administrator of UKnowledge. For more information, please contact UKnowledge@lsv.uky.edu. 


\title{
A Screen of FDA-Approved Drugs Identifies Inhibitors of Protein Tyrosine Phosphatase 4A3 (PTP4A3 or PRL-3)
}

\author{
Digital Object Identifier (DOI) \\ https://doi.org/10.1038/s41598-021-89668-5
}

\section{Notes/Citation Information}

Published in Scientific Reports, v. 11, issue 1, article no. 10302.

(C) The Author(s) 2021

This article is licensed under a Creative Commons Attribution 4.0 International License, which permits use, sharing, adaptation, distribution and reproduction in any medium or format, as long as you give appropriate credit to the original author(s) and the source, provide a link to the Creative Commons licence, and indicate if changes were made. The images or other third party material in this article are included in the article's Creative Commons licence, unless indicated otherwise in a credit line to the material. If material is not included in the article's Creative Commons licence and your intended use is not permitted by statutory regulation or exceeds the permitted use, you will need to obtain permission directly from the copyright holder. To view a copy of this licence, visit https://creativecommons.org/licenses/by/4.0/.

\section{Authors}

Dylan R. Rivas, Mark Vincent C. Dela Cerna, Caroline N. Smith, Shilpa Sampathi, Blaine G. Patty, Donghan Lee, and Jessica S. Blackburn 

identifies inhibitors of protein tyrosine phosphatase 4A3 (PTP4A3 or PRL-3)

\author{
Dylan R. Rivas ${ }^{1,5}$, Mark Vincent C. Dela Cerna ${ }^{2,5}$, Caroline N. Smith ${ }^{1}$, Shilpa Sampathi ${ }^{1}$, \\ Blaine G. Patty ${ }^{1}$, Donghan Lee ${ }^{2,3 凶}$ \& Jessica S. Blackburn ${ }^{1,4 凶}$
}

Protein tyrosine phosphatase 4A3 (PTP4A3 or PRL-3) is highly expressed in a variety of cancers, where it promotes tumor cell migration and metastasis leading to poor prognosis. Despite its clinical significance, small molecule inhibitors of PRL-3 are lacking. Here, we screened 1443 FDA-approved drugs for their ability to inhibit the activity of the PRL phosphatase family. We identified five specific inhibitors for PRL-3 as well as one selective inhibitor of PRL-2. Additionally, we found nine drugs that broadly and significantly suppressed PRL activity. Two of these broad-spectrum PRL inhibitors, Salirasib and Candesartan, blocked PRL-3-induced migration in human embryonic kidney cells with no impact on cell viability. Both drugs prevented migration of human colorectal cancer cells in a PRL-3 dependent manner and were selective towards PRLs over other phosphatases. In silico modeling revealed that Salirasib binds a putative allosteric site near the WPD loop of PRL-3, while Candesartan binds a potentially novel targetable site adjacent to the $\mathrm{CX}_{5} \mathrm{R}$ motif. Inhibitor binding at either of these sites is predicted to trap PRL-3 in a closed conformation, preventing substrate binding and inhibiting function.

Phosphatases work in concert with kinases to control phosphorylation of proteins, lipids, and other macromolecules to regulate many cellular processes. Consequently, dysregulated phosphorylation is a hallmark of cancer and multiple other diseases. Kinases catalyze phosphorylation events and have been drug targets for decades in cancer research. The critical roles of phosphatases in oncogenesis and cancer progression are just now beginning to be appreciated, and interest has grown in developing phosphatase inhibitors. More than thirty potential oncogenic phosphatases have now been identified, with roles in cellular proliferation, differentiation, migration, and angiogenesis, among others ${ }^{1}$. Protein tyrosine phosphatases (PTPs), in particular, have emerged as central regulators of cancer development and progression, with their increased activity correlated to enhanced tumor formation in mouse models and worse prognosis in patients ${ }^{2-5}$.

Members of the phosphatase of regenerating liver (PRL) family, also known as the Protein Tyrosine Phosphatase 4A (PTP4A) family, are dual specificity phosphatases that can act on both tyrosine and serine/threonine residues $^{6}$. While their physiologic cellular functions are largely unknown, the PRL family has been repeatedly shown to be involved in cancer progression. In particular, PRL-3 is a well-defined biomarker of metastasis in multiple cancer types, including melanoma, colorectal, and ovarian cancer, where PRL-3 expression is significantly higher in metastatic lesions compared to the primary tumor site ${ }^{7-15}$. In a comprehensive study of 151 patient samples across eleven common human tumors types, PRL-3 protein expression was upregulated in $80.6 \%$ of tumor samples compared to matched normal tissue ${ }^{16}$. High PRL-3 expression has also been associated with worse prognosis in human leukemia, breast, gastric, ovarian, and colorectal cancers ${ }^{9,17-20}$. Additionally, the cellular function of PRL-3 in cancer progression is now well-documented experimentally. PRL-3 overexpression in tumors results in inhibition of apoptosis, promotion of epithelial to mesenchymal transition (EMT), and enhanced migration. Additionally, PRL-3 overexpression in mouse models resulted in accelerated tumor formation and increased metastasis across a variety of tumor types ${ }^{21-24}$. Conversely, PRL-3 loss has been shown

${ }^{1}$ Department of Molecular and Cellular Biochemistry, University of Kentucky, Lexington, KY 40536, USA. 'Department of Biochemistry and Molecular Genetics, University of Louisville, Louisville, KY 40202, USA. ${ }^{3}$ Department of Medicine, James Graham Brown Cancer Center, Louisville, KY 40202, USA. ${ }^{4}$ Markey Cancer Center, Lexington, KY 40536, USA. ${ }^{5}$ These authors contributed equally: Dylan R. Rivas and Mark Vincent C. Dela Cerna. ${ }^{\square}$ email: donghan.lee@louisville.edu; jsblackburn@uky.edu 
to prevent tumor growth and metastasis in several in vivo models ${ }^{25-27}$. In one example, PRL-3 loss resulted in $50 \%$ less tumor formation in a colitis-associated colorectal cancer model ${ }^{28}$.

The other PRL family members, PRL-1 and PRL-2, share a high degree of sequence homology to PRL-3 and may possess similar functions. Like PRL-3, both PRL-1 and PRL-2 prevent contact-mediated growth inhibition, increase tumor growth, and enhance cell migration and invasion ${ }^{29-33}$. Additionally, high PRL-1 and PRL-2 expression has been reported in a variety of cancer types including cervical, hepatic, and breast cancers ${ }^{34-36}$. Although less studied than PRL-3, data indicate that PRL-1 and PRL-2 overexpression increases metastasis in mouse models, while their loss decreases tumor cell migration and invasion. Together these results demonstrate the importance of the PRL family both in tumor formation and cancer progression, which has made them attractive therapeutic targets.

Currently, clinically available PRL inhibitors are lacking. This is in large part due to several significant challenges associated with small molecule inhibition of the PRL family, including the high level of homology between the PRLs, which makes targeting individual PRLs difficult, and conservation of the active site among PRLs and other tumor suppressive tyrosine phosphatases, such as PTEN ${ }^{37,38}$. Additionally, the PRL active site is shallower, wider, and more hydrophobic than other phosphatases, making design of PRL-specific inhibitors more challenging. In spite of these obstacles, several groups have identified or developed PRL-specific inhibitors including Thienopyridone, JMS-053, Compound 43, and Analog $3^{39-43}$. While these compounds exhibit anti-cancer effects in vitro and in mouse xenograft studies, difficulties in formulation have thus far prevented further development into clinical and therapeutic agents. Promisingly, a humanized PRL-3 antibody was recently developed that prevents growth of PRL-3 expressing tumors, while not targeting PRL-3-negative tissues. This antibody is currently in phase II clinical trials for patients with advanced solid tumors that have failed standard therapy outcomes have yet to be measured, and this drug will take some time to be widely available if it is proven safe and effective $e^{16,44}$.

To address the immediate need for PRL-3 inhibitors in the clinic, we screened a library of 1443 FDA approved drugs for their ability to modulate the phosphatase activity of PRLs. We found one selective inhibitor of PRL-2, five selective inhibitors of PRL-3, and nine potent inhibitors of the PRL family. Two drugs from the latter group, Salirasib and Candesartan, prevented PRL-3 mediated cell migration in PRL-3 overexpressing human embryonic kidney (HEK) cells without impacting cell viability. Furthermore, these compounds were able to inhibit migration in a colorectal cancer cell line that expressed endogenously high levels of PRL-3. In silico docking of the drugs to PRL-3 showed that Salirasib binds to PRL-3 in the same site proposed for the research-grade PRL-3 inhibitor, JMS-053, while Candesartan binds a secondary site in PRL-3 that has not previously been targeted. These drugs appear to function allosterically by locking PRLs in the closed confirmation. Together, our results indicate that it may be possible to repurpose FDA-approved drugs to block PRL-3 activity in human cancer cells. These drugs may also provide some insight into the structures of compounds that are best able to selectively target PRL-3 and can be used as chemical probes to uncover biological functions of PRL-3.

\section{Results}

Identification of FDA-approved drugs that inhibit PRL activity. Phosphatase activities of recombinantly expressed PRL-1, PRL-2, and PRL-3 were evaluated based on their ability to hydrolyze the synthetic substrate, phospho-tyrosine analog 6,8-Difluoro-4-methylumbelliferyl phosphate (DiFMUP). DiFMUP hydrolysis could be significantly inhibited by three research-grade PRL inhibitors: the rhodamine-derivative PRL Inhibitor $\mathrm{I}^{45}$, Analog $3^{41}$, and Thienopyridone ${ }^{40}$ (Supplemental Fig. 1). Similar to prior reports, Thienopyridone was most effective, blocking PRL activity by 95\%, and was used as a positive control for PRL inhibition throughout the rest of our studies. We next screened 1,433 FDA-approved drugs for their ability to inhibit PRL dephosphorylation of DiFMUP. Phosphatase activity of the PRL after drug treatment ranged from 0 to $150 \%$ of the dimethylsulfoxide (DMSO) control. Fifty-three compounds that emitted fluorescence autonomously were excluded due to the potential interference with data analysis. The mean phosphatase activity across the remaining 1,380 drugs was calculated for each PRL, and a compound was considered a hit in this assay when the drug reduced the phosphatase activity of the PRL to three standard deviations lower than the mean phosphatase activity (Supplemental Table 1). The DiFMUP assay was then repeated on the initial hits, which confirmed nine broad inhibitors that blocked the phosphatase activity of PRL-1, PRL-2, and PRL-3 by more than $80 \%$ (Fig. 1a, Supplemental Fig. 2). Additionally, there was a single drug that preferentially inhibited PRL-2, and four drugs that preferentially inhibited PRL-3 over other PRLs (Fig. 1b, c).

All broad inhibitors blocked PRL phosphatase activity in a dose dependent manner, between $1 \mathrm{nM}$ and 100 $\mu \mathrm{M}$. The broad PRL inhibitors loosely separated into those with high $\mathrm{IC}_{50}$ values (greater than $1 \mu \mathrm{M}$ ), and low $\mathrm{IC}_{50}$ values (less than $1 \mu \mathrm{M}$ ) for PRL-1, PRL-2 and PRL-3 (Fig. 2a, b, Supplemental Fig. 3). Additionally, the PRL-3 specific inhibitors were also able to selectively inhibit PRL-3 phosphatase activity at a significantly lower $\mathrm{IC}_{50}$ than PRL-1 and PRL-2 $(p<0.0001)$, although they did inhibit these phosphatases to an extent (Fig. $2 \mathrm{c}, \mathrm{d}$, Supplemental Fig. 4). On average the broad PRL inhibitors had an $\mathrm{IC}_{50}$ value threefold lower than the specific PRL-3 inhibitors (Table 1), so we focused on the broad PRL inhibitors. The specific PRL-2 inhibitor, ABT-199, is also a potent inhibitor of $\mathrm{Bcl}-2^{46}$. Due to the probable associated toxicity at the dose of drug needed for effective PRL-2 inhibition, this drug was not pursued further.

A subset of compounds is non-toxic in HEK293T cells at doses that are inhibitory to PRL-3. Drugs were next tested for off-target effects on the cell viability at the $\mathrm{IC}_{50}$ of PRL-3 inhibition. The immortalized human embryonic kidney cell line 293T (HEK293T) expresses very low levels of PRL-3 and any changes in viability with drug treatment would be due to off-target toxcity. Cells were treated with doses of drug and analyzed by an MTT (3-(4-5-dimethylthiazol-2-yl)-2,5-diphenyltetrazolium bromide) assay, which measures cellular metabolic activity as a is a read-out of the cell viability. Salirasib, Vitamin B12 and the positive con- 
a

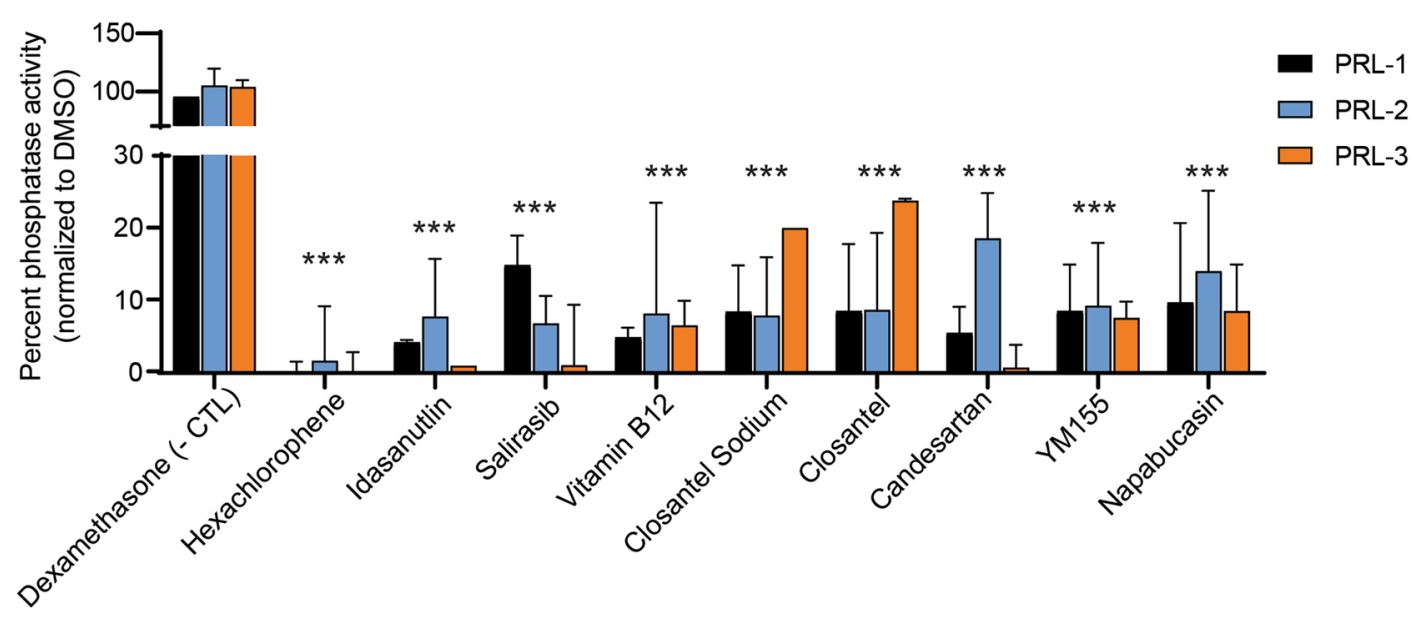

b

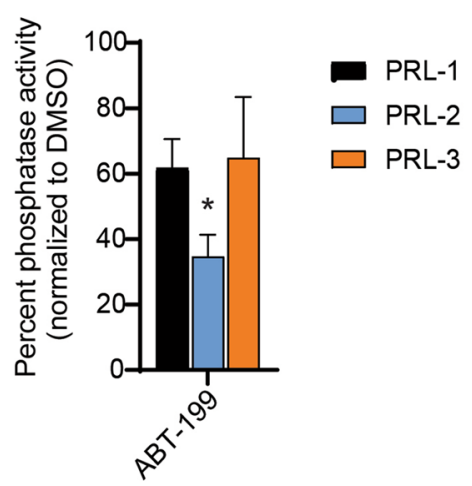

C

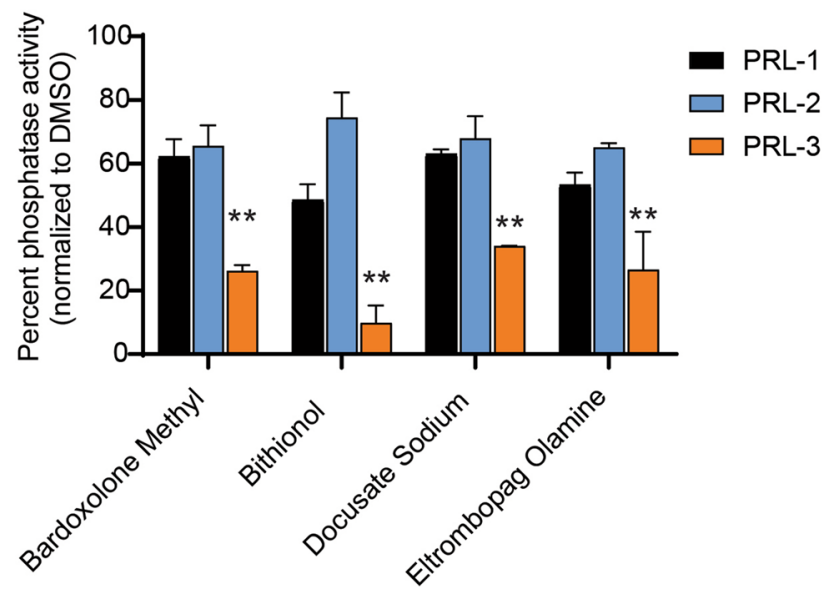

Figure 1. Multiple FDA-approved compounds inhibit the phosphatase activity of PRL proteins. (a) Validation of drugs that were positive hits in the initial FDA-drug screen and significantly reduced PRL phosphatase activity across multiple tests. The FDA-approved drug Dexamethasone, which has no effect on PRL-3 phosphatase activity, was used as a negative control (- CTL). (b) ABT-199 demonstrated selectivity in inhibiting PRL-2. (c) Validation of PRL-3 specific inhibitors. All assays were run at a final PRL protein concentration of $2.5 \mu \mathrm{M}$, a drug concentration of $40 \mu \mathrm{M}$, and DiFMUP concentration at the previously reported $\mathrm{K}_{\mathrm{M}}$ of the protein. Bars represent the average phosphatase activity of the initial screen plus two additional independent experiments, in triplicate. Error bars represent standard deviation. ${ }^{*} p<0.05,{ }^{* *} p<0.001,{ }^{* *} p<0.0001$ by either two-way ANOVA with Dunnet's correction (a) or one-way ANOVA with Tukey's HSD (b, c).

trol for PRL-3 inhibition, Thienopyridone, had no effect on the viability at drug doses ranging from 1 to $100 \mu \mathrm{M}$ (Fig. 3a) after 16 hours of incubation with the compounds. Candesartan showed insignificant effect on viability up to the concentration of $50 \mu \mathrm{M}$ but viability dropped significantly at $100 \mu \mathrm{M}$, indicating potential toxicity of the compound at concentrations well above the $\mathrm{IC}_{50}$ for PRL-3 inhibition. Hexachlorophene and Closantel increased MTT absorbance in 293 T cells (Fig. 3b). Finally, Idasanutlin, YM155, and Napabucasin decreased viability of 293T cells (Fig. 3c), most likely due to these drugs having a known higher affinity for proteins involved in apoptotic pathways ${ }^{47,48}$ and potentially other off-target effects. As the ultimate goal of this study is to identify drugs that could be used as anti-cancer therapy in PRL-3 expressing tumors, we eliminated drugs that enhanced cell viability, and focused on those that decreased or caused no change in cell viability.

FDA-approved drugs blocked PRL-3 induced cell migration in human cancer cells. PRL-3 is well-established to promote cell migration. Scratch assays were used to test whether broad PRL inhibitors would alter the migratory phenotype of PRL-3 overexpressing HEK293T cells and human colorectal cancer cells that have endogenously high levels of PRL-3. Transfection of a PRL-3 expression vector increased PRL-3 expression in HEK293T cells by fourfold and enhanced cell migration by $40 \%$ ( $p=0.02$, Fig. 4a, Supplemental Fig. 5). Empty vector and PRL-3 transfected cells were then treated with the broad PRL inhibitors at concentrations that were previously found to not affect cell viability by more than $25 \%$. Treatment with two of the drugs, Salirasib and Candesartan, resulted in a significant inhibition of cellular migration in PRL-3 overexpressing cells, compared to DMSO treatment (Salirasib, $p=0.03$ and Candesartan $p=0.001$, Fig. $4 \mathrm{~b}, \mathrm{c}$ ) with no corresponding reduction in migration in control cells. These data indicate that these drugs can directly inhibit PRL-3 to impact 
a

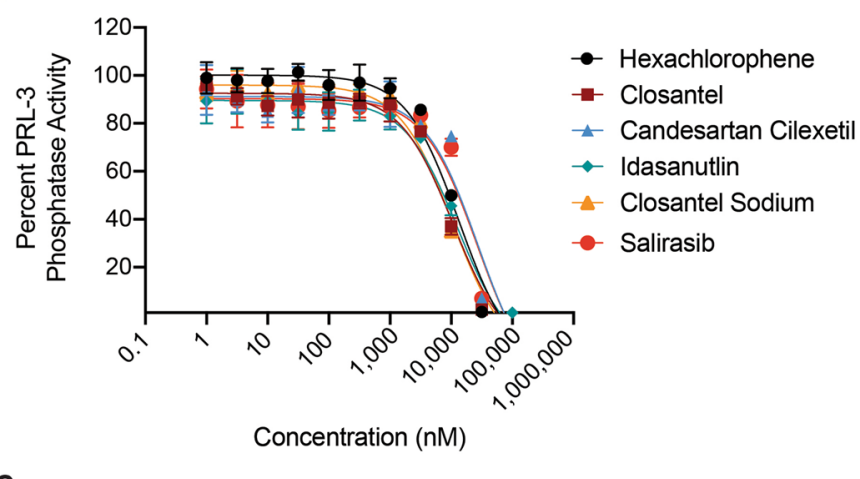

C

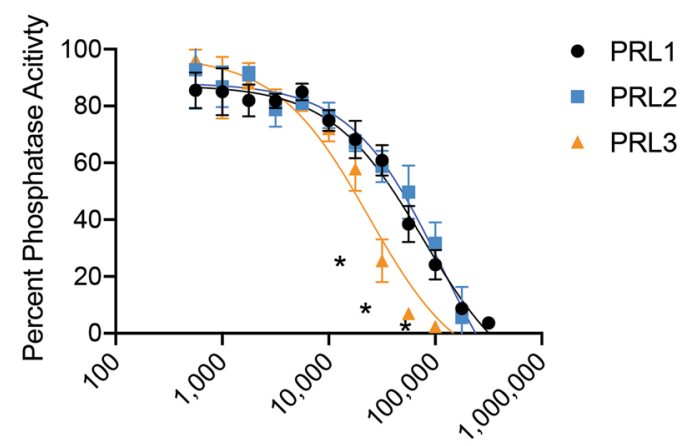

Bardoxolone (nM) b

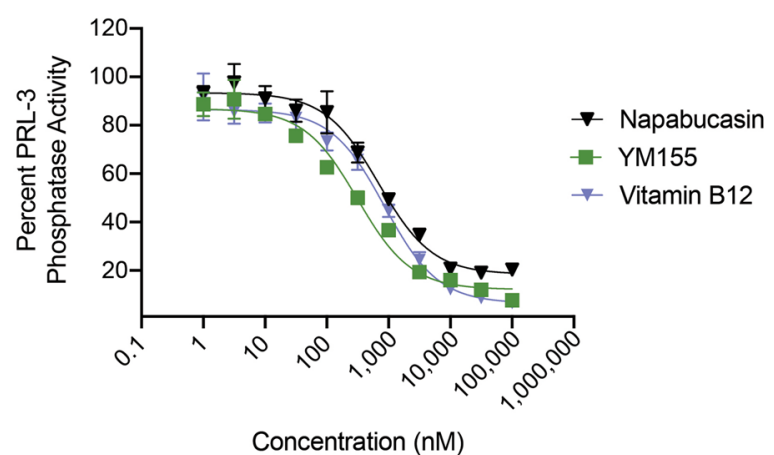

d

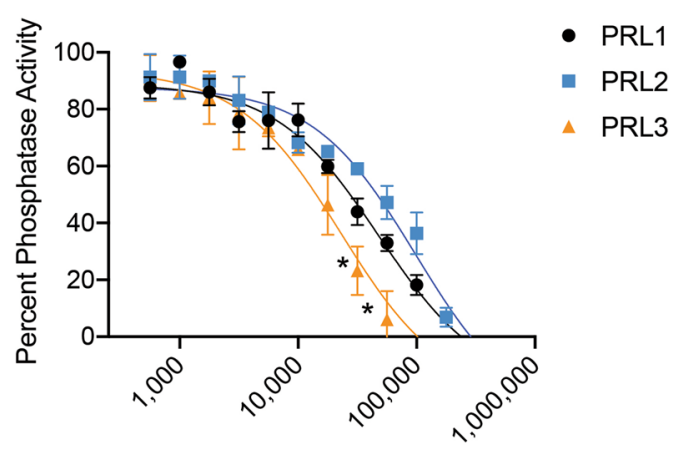

Eltromobag $(\mathrm{nM})$

Figure 2. Dose dependent inhibition of PRL family members with FDA-approved drugs. Dose response curves showing DiFMUP phosphatase activity of PRL-3 when treated with doses ranging between $1 \mathrm{nM}$ and $100 \mu \mathrm{M}$ of each drug. Inhibition of PRL-3 phosphatase activity by the broad PRL family inhibitors are shown, and drugs are subdivided into those with (a) high $\mathrm{IC}_{50}$ values and (b) low $\mathrm{IC}_{50}$ values. A subset of compounds preferentially inhibited PRL-3, including (c) Bardoxolone and (d) Eltromobag. All assays utilized a final protein concentration of $2.5 \mu \mathrm{M}$ with DiFMUP concentration at the $\mathrm{K}_{\mathrm{M}}$ of the protein, and were run in technical duplicates in three independent experiments. Data represent mean phosphatase activity and error bars represent standard deviation between assays. ${ }^{\star} p<0.01$ comparing PRL-3 phosphatase activity to that of PRL-1 and PRL-2 at the indicated drug dose, using a two-way ANOVA with Dunnet's correction.

\begin{tabular}{|c|c|c|c|}
\hline & PRL-1 & PRL-2 & PRL-3 \\
\hline \multicolumn{4}{|l|}{ Broad PRL inhibitors } \\
\hline Candesartan & $69 \pm 8$ & $80 \pm 15$ & $28 \pm 4$ \\
\hline Closantel & $17 \pm 3$ & $14 \pm 5$ & $11 \pm 0.5$ \\
\hline Closantel Sodium & $22 \pm 8$ & $18 \pm 1$ & $10 \pm 0.4$ \\
\hline Hexachlorophene & $31 \pm 4$ & $35 \pm 10$ & $13 \pm 1$ \\
\hline Idasanutlin & $20 \pm 3$ & $16 \pm 2$ & $13 \pm 0.4$ \\
\hline Napabucasin & $0.4 \pm 0.2$ & $1.1 \pm 0.3$ & $0.7 \pm 0.1$ \\
\hline Salirasib & $53 \pm 5$ & $118 \pm 22$ & $27 \pm 3$ \\
\hline Vitamin B12 & $0.7 \pm 0.2$ & $0.9 \pm 0.2$ & $0.9 \pm 0.1$ \\
\hline YM155 & $0.3 \pm 0.1$ & $0.7 \pm 0.1$ & $0.3 \pm 0.1$ \\
\hline \multicolumn{4}{|c|}{ PRL-3 specific inhibitors } \\
\hline Bardoxolone & $85 \pm 43$ & $136 \pm 75$ & $24 \pm 5^{*}$ \\
\hline Bithionol & $79 \pm 25$ & $115 \pm 34$ & $33 \pm 2^{*}$ \\
\hline Docusate Sodium & $94 \pm 26$ & $140 \pm 44$ & $43 \pm 5^{\star}$ \\
\hline Eltromobag & $56 \pm 10$ & $105 \pm 19$ & $26 \pm 11^{*}$ \\
\hline Eltromobag Olamine & $61 \pm 15$ & $166 \pm 22$ & $27 \pm 4^{*}$ \\
\hline Embelin & $114 \pm 16$ & $124 \pm 60$ & $52 \pm 9^{*}$ \\
\hline
\end{tabular}

Table 1. Calculated $\mathrm{IC}_{50}$ values in $\mu \mathrm{M}$ from broad and specific inhibitors of PRL phosphatase activity. ${ }^{*} p<0.05$ between IC50 values for PRL-3 and PRL-1 and PRL-2 by one-way ANOVA with Tukey's HSD. 

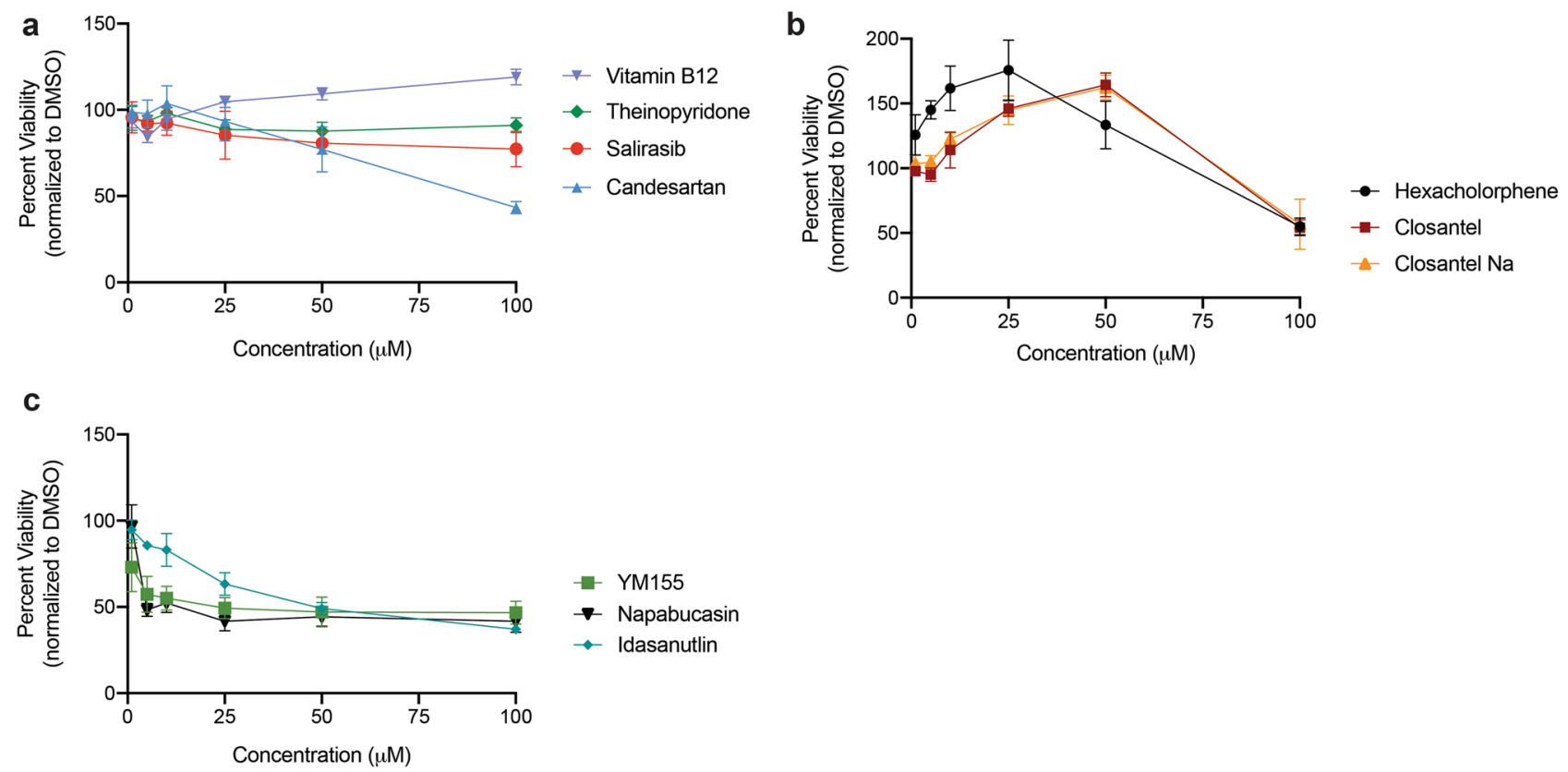

Figure 3. Salirasib and Candesartan are non-toxic PRL inhibitors. HEK293T cells were cultured with drugs at a range of concentrations and viability was assessed after $16 \mathrm{~h}$. Drugs were sub-divided into those that were (a) minimally toxic, (b) caused increased viability, and (c) were highly toxic. Viability was measured as cellular reduction potential using MTT dye, and normalized to $1 \%$ DMSO control. Assays were run in triplicate in three independent experiments. Data represent mean viability and error bars represent standard deviation between assays.

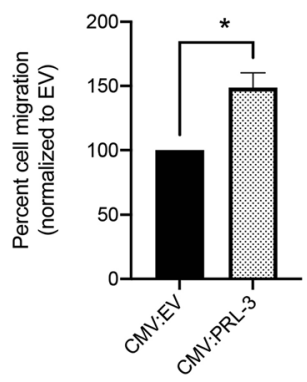

b

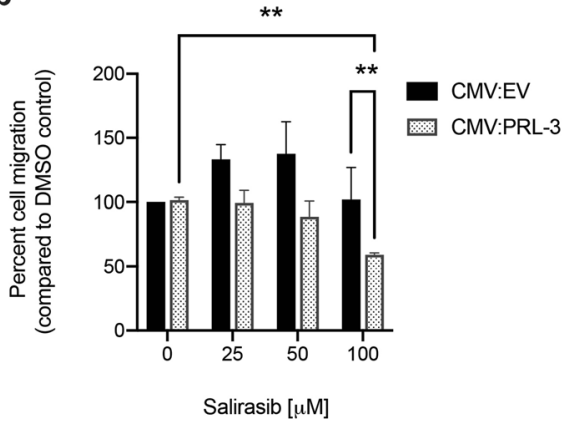

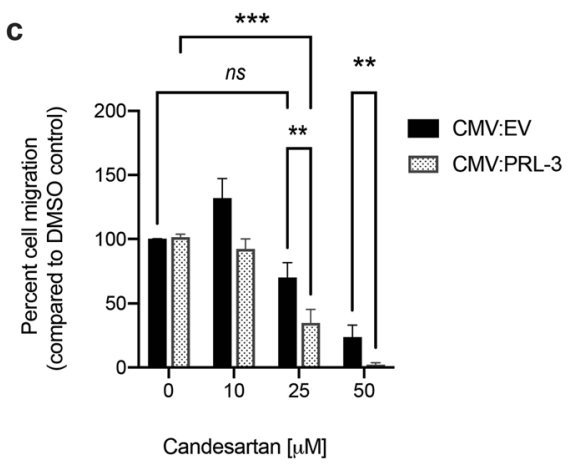

Figure 4. Salirasib and Candesartan inhibit PRL-3 induced cell migration in HEK293T cells. (a) Quantification of cell migration of HEK293T cells transfected with a either PRL-3 expression vector or empty vector control. Cells from (a) were treated with either (b) Salirasib or (c) Candesartan at the doses indicated. The zero drug dose was a $1 \%$ DMSO control, and each data set was compared to the DMSO control group within that data set. Both drugs inhibited cell migration to a greater extent in the PRL-3 overexpressing cells, compared to empty vector $(\mathrm{EV})$ control. ${ }^{\star} p=0.089$ using one tailed student $\mathrm{t}$ test with Holm Sidak correction, ${ }^{\star *} p=0.03$, ${ }^{* * *} p=0.001, n s=$ no significant difference, using a two-way ANOVA with Dunnett's correction.

cellular migration. Other drugs, including Idasanutlin, YM155, and Napabucasin had less significant effects on the migration of PRL-3 expressing cells, compared to control cells, or impacted migration in both PRL-3 overexpressing and control cells equally, and were therefore excluded from further analysis (Supplemental Fig. 6). Neither Salirasib nor Candesartan had a negative effect on the activity of 18 other phosphatases (Supplemental Table 2).

Next, we tested whether Salirasib and Candesartan could decrease cellular migration in human colorectal cancer cells that express high levels of endogenous PRL- $3^{12,22}$. We found that both drugs significantly inhibited the migration of the HCT116 and SW 480 cells by $>30 \%$ ( $p=0.03$, Fig. 5a, b). This drug-induced change in migration was not due to any significant change in proliferation or apoptosis rates in the cells at $24-48 \mathrm{~h}$ post-treatment (Supplemental Fig. 7). Although direct PRL-3 substrates are largely unknown, the ERK signaling pathway has previously been shown to be affected by PRL-3 activity in a variety of cancer cells, where it plays a role in cell 
a

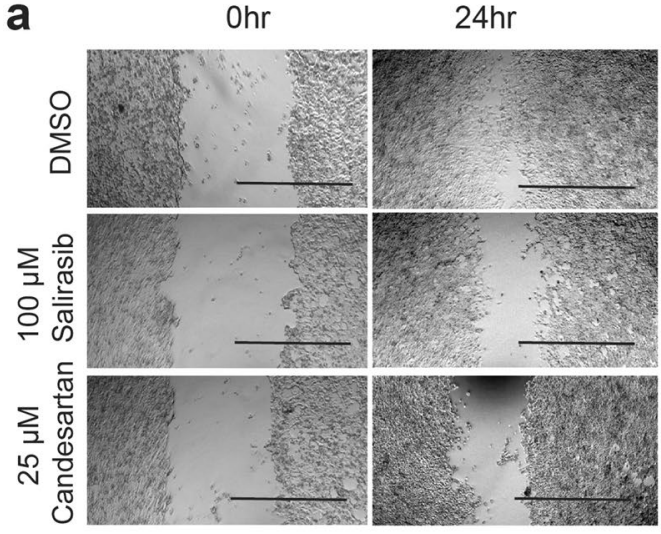

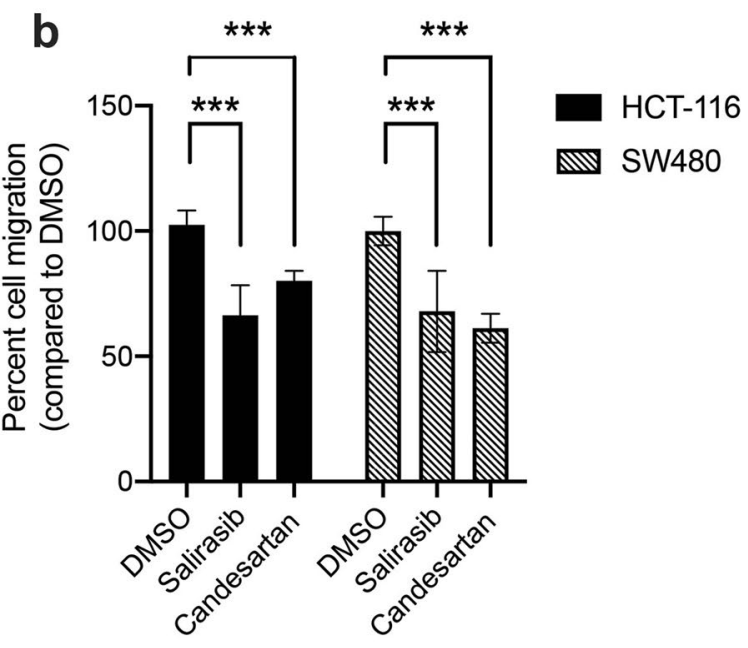

C

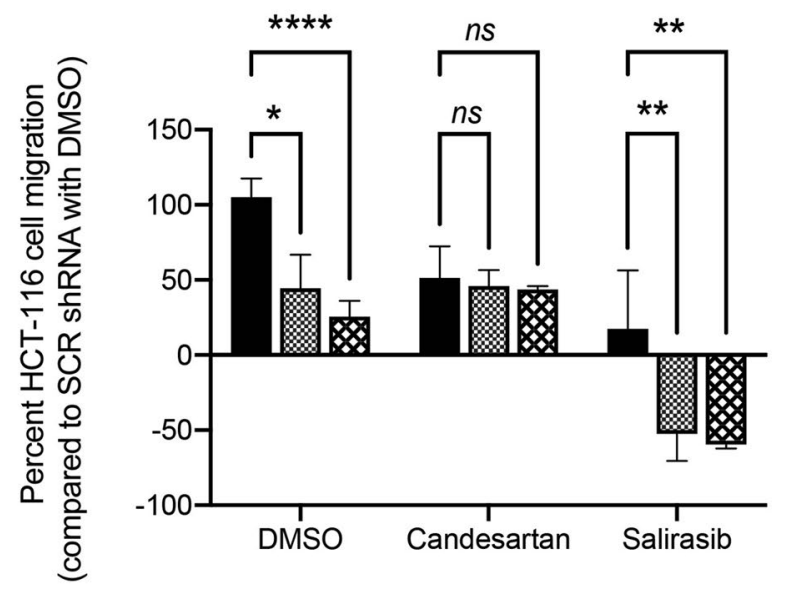

Figure 5. Salirasib and Cadnesartan inhibit PRL-3 mediated cell migration in human colorectal cancer cells. (a) Representative images of migration of the human colorectal cancer cell line HCT116 when treated with 1\% DMSO, $100 \mu \mathrm{M}$ Salirasib or $25 \mu \mathrm{M}$ Candesartan. (b) Quantification (a) across three independent experiments, in triplicate, in both HCT116 and SW480 cells. Migration was measured by quantifying the empty area in the image of the entire well at 0 and $24 \mathrm{~h}$ post-scratch using Image and calculating the difference. Percent migration was measured as the area migrated $24 \mathrm{~h}$ after scratch normalized to the area migrated by DMSO control. (c) Migration of HCT116 cells that were transfected with either scrambled or PRL-3 shRNA and then treated with 1\% DMSO, $100 \mu \mathrm{M}$ Salirasib or $25 \mu \mathrm{M}$ Candesartan. Data were quantified as above, except that all data sets were normalized to the Scrambled shRNA transfected cells treated with DMSO. Negative values indicate that the empty area was larger at $24 \mathrm{~h}$ than the 0 timepoint. All assays were run in duplicate wells, in 3 independent experiments. Bars indicate the mean percent cell migration, and error bars represent standard deviation between assays. ${ }^{* * *} p=0.026$ using one-way ANOVA with Tukey's HSD, ${ }^{\star} p=0.039,{ }^{* * *} p=0.0021,{ }^{* *} p=0.02, n s=$ not significant, analyzed by a two-way ANOVA with Dunnett's correction.

migration and invasion ${ }^{49,50}$. Both Salirasib and Candesartan decreased phospho-ERK levels in HCT116 cells to a similar extent as the research grade PRL-3 inhibitor, Thienopyridone (Supplemental Fig. 8).

We next transfected HCT116 cells with shRNA that we have previously found to be effective against PRL- $3^{51}$, producing a $>50 \%$ reduction in PRL-3 protein (Supplemental Fig. 5), and saw the expected decrease in colorectal cancer cell migration (Fig. 5c, Supplemental Fig. 9). Candesartan treatment of cells with PRL-3 knock-down showed no additional impact on migration, suggesting that this drug largely acts through PRL-3 to reduce cell migration (Fig. 5c). Interestingly, colorectal cancer cells treated with Salirasib in combination with PRL-3 shRNA had significantly reduced migration compared to control shRNA, with the scratch widening after $24 \mathrm{~h}$, indicating some die-off of PRL-3 shRNA/Salirasib treated cells that is not present in the control shRNA/Salirasib treated cells (Supplemental Fig. 9). These data indicate that Salirasib is not as specific for PRL-3 as Candesartan. As a known Ras inhibitor, Salirasib may target other important pathways in colorectal cancer cells that can synergize with PRL-3 knockdown to impact both cell viability and migration.

In silico docking of Salirasib and Candesartan on PRL-3. Due to the lack of currently available structure of monomeric PRLs in complex with any inhibitor, inhibitor binding sites are largely unknown. Attempts by other groups to obtain co-crystals of PRL-3 with inhibitors have thus far been unsuccessful. Molecular dock- 

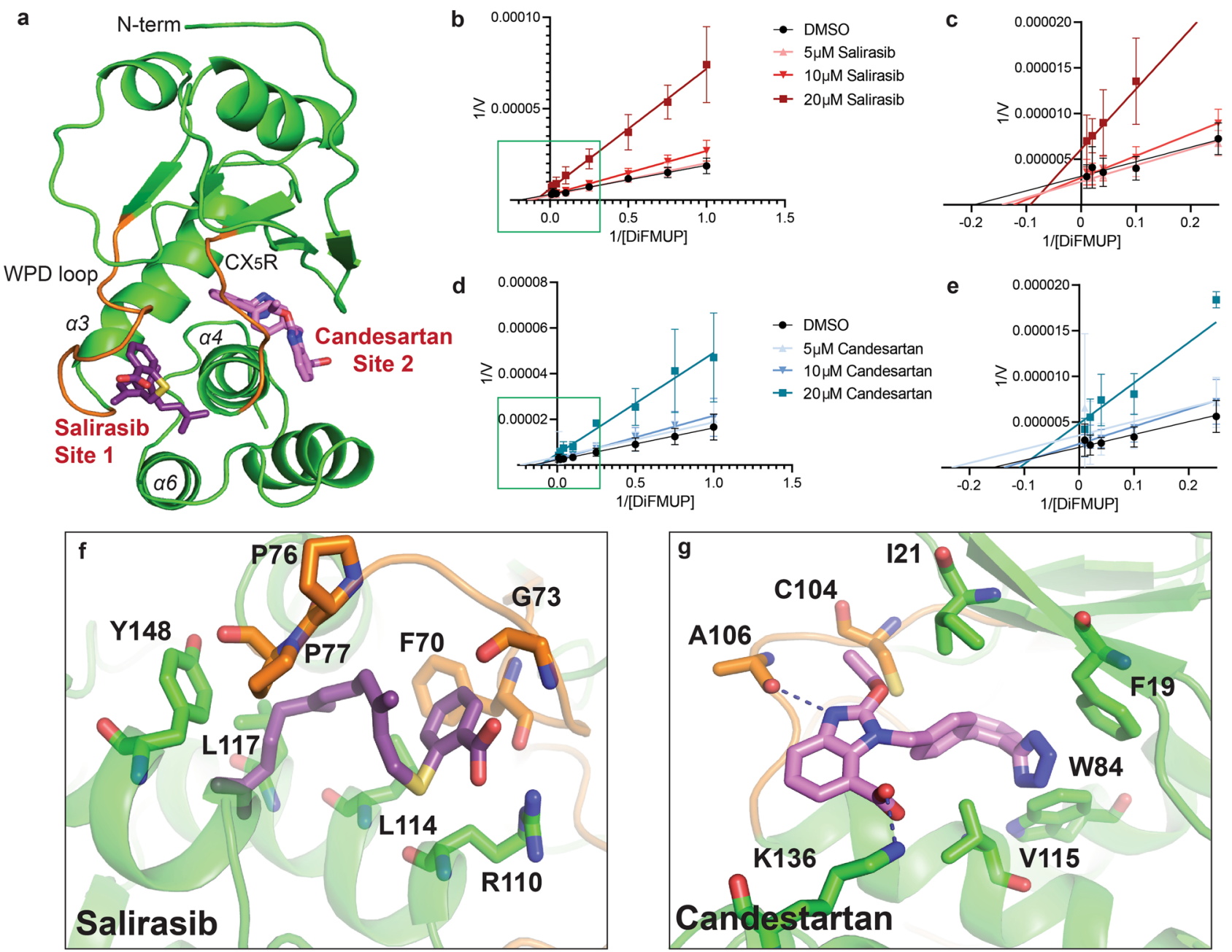

Figure 6. Molecular docking experiments reveal that top hits from screening FDA-approved drugs bind to allosteric sites. (a) Representive compounds, Salirasib and Candesartan, are shown bound to two identified allosteric sites adjacent to the active site looops (orange) in the closed conformation of PRL-3 (PDB: 2MBC). Lineweaver-burk plots indicate that Salirasib $(\mathbf{b}, \mathbf{c})$ and Candesartan $(\mathbf{d}, \mathbf{e})$ are non-competitive inhibitors to the substrate, supporting the docking results. Graphs in (c) and (e) are insets of the boxed areas in (b) and (d). Assays were run with technical duplicates and performed in 3 independent replicates. Error bars represent standard deviation between assays. Proposed binding modes of Salirasib (f) and Candesartan (g) on PRL-3, with residues in close proximity to the bound molecule identified.

ing was therefore employed to identify the potential binding sites of the drugs used in our assay, including the potent inhibitors Salirasib and Candesartan, and to further understand their mechanisms of action. The docking simulations show that, expect for Hexachlorophene, all of the drugs bind preferentially (with lower binding energies) to the closed conformation of PRL-3 (Supplemental Table 3, Supplemental Fig. 10). In the closed form, two major binding sites were identified: Site 1, which is flanked by the WPD loop and helices $\alpha 3, \alpha 4$, and $\alpha 6$ and has been previously predicted to be an allosteric binding site for the research-grade PRL-3 inhibitor, JMS- $053^{11}$, and Site 2, a potential secondary allosteric site adjacent to the PRL-3 $\mathrm{CX}_{5} \mathrm{R}$ motif and bound by helix $a 4$ and the $\beta$-sheets (Fig. 6a). Lineweaver-Burke analyses show that both Candesartan and Salirasib reduce apparent $V_{\max }$ but do not affect the $\mathrm{K}_{\mathrm{M}}$ and are non-competitive inhibitors to the DiFUMP substrate (Fig. 6b-e). Therefore, both inhibitors bind sites other than the active site, consistent with our in silico models indicating that both compounds bind to potential allosteric sites.

In silico modeling suggests that Salirasib binds in the allosteric Site 1 with a free energy of binding of -10.46 $\mathrm{kcal} / \mathrm{mol}$. This pocket is lined with hydrophobic residues that likely stabilizes the farnesyl tail of the drug. The salicylic acid group of Salirasib is then free to form hydrogen bonding, in this case with the backbone amide and carbonyl groups of G73 (Fig. 6f), likely stabilizing PRL-3 in a closed conformation. Candesartan binds at the potentially new allosteric Site 2 with an estimated free energy of binding of $-10.26 \mathrm{kcal} / \mathrm{mol}$ for the closed site. In particular, it fits in a shallow binding pocket lined with hydrophobic residues, similar to Site 1, and can form electrostatic interactions with PRL-3 through the sidechain of K136 and the backbone carbonyl of A106 (Fig. 6g). The low docking scores are expected due to the low molecular weight of the drugs. The drugs and the sites are also largely hydrophobic. Regardless, these docking simulations show a potential mechanism for PRL-3 inhibition 
by Salirasib and Candesartan. Additionally, Site 2 offers an additional targetable site that to our knowledge has not been pursued specifically in the past.

As a validation of these results, drugs that were used as controls in experimental work were docked. Dexamethasone was used as a negative control, while the rhodanine derivative PRL-3 Inhibitor was used as a positive control in the drug screen. In both conformations, dexamethasone was the lowest ranked, while PRL-3 Inhibitor is among the higher ranked. Finally, a farnesyl tail was docked to verify binding of the farnesyl derivative Salirasib. Blind docking identified the same binding pocket in Site 1 (Supplemental Fig. 11).

\section{Discussion}

PRL-3 is highly expressed in many cancer types and is a proven oncoprotein. It has well-established roles in tumor cell invasion and metastasis, and data suggests it may also be involved in cancer cell proliferation and drug resistance ${ }^{52,53}$. There have been increasing efforts to identify PRL-3 inhibitors. A screen of the Korea Chemical Bank identified the rhodanine derivatives CG-707 and BR-1 as PRL inhibitors, but further analysis showed these to be fairly nonspecific ${ }^{45}$. Screening of the Roche chemical library identified Thienopyridone as a PRL-3 inhibitor that reduced tumor growth by interfering with cell adhesion, although it is too toxic for in vivo use ${ }^{40}$. Further analysis of Thienoypridone, relying on active site mimicry and in silico structural modeling, led to the discovery of an additional PRL-3 inhibitor, Analog $3^{41}$. A subsequent structural activity relationship study identified iminothienopyridone 13 (JMS-053), an analog with lower toxicity and increased potency than Thienopryidone ${ }^{42}$. This compound is notable because it is the first PRL-3 small molecule inhibitor used in vivo, where it inhibited cancerous cellular growth in a multi-drug resistant ovarian xenograft model. However, the solubility of JMS-053 is poor, and it requires further study to determine pharmacokinetics, toxicity, and efficacy in humans. Finally, a specific PRL-3 binding antibody has been developed, which is currently the only inhibitor capable of selectively targeting PRL-3 over other family members and is capable of preventing tumor growth using in vivo model of both hepatic and gastric cancers ${ }^{16,44}$. Although antibody treatments are an effective strategy for targeting proteins in a variety of diseases, the associated costs are significantly higher than using a small molecule inhibitor, and this antibody will also require clinical trials for safety and efficacy. Although efforts are underway to improve each of these compounds and biologics, it can take many years to bring a drug from development to the clinic.

Through our screen of $>1400$ FDA-approved drugs, we identified one compound, Candesartan, that significantly reduced PRL phosphatase activity. Candesartan is FDA-approved as an angiotensin receptor blocker to treat high-blood pressure, and has not previously been used as an anti-cancer therapy. Angiotensin receptors are G-protein coupled receptors that are typically involved in transmitting vasoconstrictive stimuli in in blood vessels, and are variably expressed in cancer cells. Interestingly, Candesartan has been found to interact with the proteins Plekhj1, Sh2b3, and DotL1, all of which are involved in various signaling pathways in cancer cells. Our data indicate that Candesartan blocks PRL-3 phosphatase activity, can revert PRL-3 induced migration in HEK293T cells, and can reduce migration of colorectal cancer cells that express high levels of PRL-3, with no impact on migration in the same cells with PRL-3 knocked down via shRNA. While these data suggest some specificity of Candesartan for PRL-3, additional studies are needed to define the other pathways and cellular responses that might be affected by this drug across different cancer types.

A second drug identified in this screen, Salirasib, is an S-farnesyl cysteine analog that functions to dislodge Ras-isoforms from their membrane anchoring sites, leading to Ras degradation. Many cancers rely on dysregulated Ras signaling for a variety of cellular processes, including cell growth and migration. While Salirasib blocked PRL-3 phosphatase activity in a cell-free system, it is difficult to ascribe the cellular functions of Salirasib strictly to its interactions with PRL-3-Salirasib treatment of colorectal cancer cells with PRL-3 knock-down appeared to die to an extent not seen with either PRL-3 shRNA or Salirasib treatment alone, suggesting multiple mechanisms of action for this drug, perhaps in synergy with PRL-3 loss. Additionally, similar to Ras, PRL-3 is farnesylated and localized to the cell membrane. PRL-3 would therefore be similarly outcompeted for membrane anchoring sites by Salirasib, suggesting that the drug may both be blocking PRL-3 activity through interaction with the protein, and causing PRL-3 mis-localization by preventing membrane binding.

The lack of available structural information of PRL-3 alone or in complex with an inhibitor makes it challenging to identify targetable residues in PRL-3 or propose binding sites for potential inhibitors. However, docking functionally validated PRL inhibitors via in silico approaches can provide useful information on PRL-3 interactions with drugs. We found that Salirasib binds to a previously identified binding pocket adjacent to the conserved WPD loop; the allosteric inhibitor JMS-053 was also proposed to bind to this site ${ }^{11}$. Candesartan binds to a secondary pocket at the opposite side of the active site, adjacent to the $\mathrm{CX}_{5} \mathrm{R}$ motif. Both of these binding sites are lined with hydrophobic residues that may allow binding of highly hydrophobic small molecules, like Candesartan $(\log P$ of 6.1$)$ and Salirasib $(\log P$ of 6.8$)$. To our knowledge, this secondary allosteric site has not been previously identified before as a binding site for inhibitors of PRL-3 and provides an alternative target site for in silico screens to identify novel molecules targeting PRL-3. Additionally, while Salirasib and Candesartan were able to inhibit PRL-3 phosphatase activity and affect cellular function, the $\mathrm{IC}_{50}$ s were in the $\mu \mathrm{M}$ range. Future work modifying these compounds may be useful to increase the strength of binding and improve their potency.

Our models also show that drug binding might be sensitive to PRL-3 conformation. The WDP loops and the $\mathrm{CX}_{5} \mathrm{R}$ motif (or P loop) harbor the catalytic residues of classical dual-specificity phosphatases. The two allosteric sites identified are located adjacent to these loops. The catalytic cycle of PRLs and other classical phosphatases are characterized by large structural rearrangements primarily in the WPD loop and surrounding region. As PRL-3 transitions to its open state, the catalytic loops move a total of about $10 \AA$ towards each other, moving the catalytic residues into close proximity. This includes the nucleophilic cysteine (P loop, C104 in PRL-3), the aspartic acid (WPD loop, D72) that acts as the general base/proton donor, and the conserved arginine (P loop, R110) that stabilizes the phosphoenzyme intermediate. Our modeling suggests that the drugs have a lower 
binding energy for the closed PRL-3 conformation - this indicates a possible mechanism of action where these small molecules trap PRL-3 in the closed conformation, preventing active site structural rearrangements, which is necessary for catalysis ${ }^{54,55}$.

Finally, targeting protein phosphatases such as PRL family members has been challenging due to high sequence similarity and the conserved active sites among protein tyrosine phosphatases. Additionally, small molecule inhibitors have little selectivity between the PRLs, since the PRL active site is wide and shallow; the most effective PRL-3 inhibitors identified in this study also targeted the entire PRL family. Allosteric inhibition of PRLs will provide an alternate strategy for the identification and development of more specific PRL-3 inhibitors. Thus far, two potential allosteric sites are identified for PRL-3 and further studies are needed to experimentally validate both sites, as well as the possibility of targeting them with small molecules. Several drugs, including Bardoxolone and Eltromobag, showed greater specificity for PRL-3 over PRL-1 and PRL-2. The properties of these drugs may be useful in informing future development of novel PRL-3 inhibitors.

\section{Methods}

Recombinant protein expression. PRL full length protein sequences fused to an N-terminal 6xHis-tag was cloned into pET28b bacterial expression vector. Proteins were expressed in One Shot BL21 Star bacteria (Invitrogen, Cat \# C601003) by induction with $0.5 \mathrm{mM}$ IPTG (Fisher Scientific, Cat \# BP175510) for $16 \mathrm{~h}$. Cells were resuspended in $10 \mathrm{ml}$ of lysis buffer [ $300 \mathrm{mM} \mathrm{NaCl}$ (VWR Cat. No. BDH9286), $20 \mathrm{mM}$ Tris pH 7.5, $10 \mathrm{mM}$ Imidazole pH 8.0 (Sigma-Aldrich I2399), 1:1000 protease inhibitor cocktail (Sigma-Aldrich Cat. No. P8465)] per gram of cell pellet and lysed using a microfluidizer (Avestin, EmulsiFlex-C5). Protein was isolated using Ni-NTA Resin (VWR, Cat \# 786-940) and eluted with $2 \mathrm{~mL}$ of elution buffer (300 mM NaCl, $20 \mathrm{mM}$ Tris pH 7.5, and $250 \mathrm{mM}$ Imidazole $\mathrm{pH}$ 8.0). Following cleavage with TEV protease, samples were reapplied to Ni-NTA column to remove uncleaved protein as well as TEV. Samples were further purified using an S200 column on a Superdex 10/300 in buffer containing $100 \mathrm{mM} \mathrm{NaCl}$ and $200 \mathrm{mM}$ HEPES pH 7.5. Purified fractions were then run on $4-20 \%$ Mini-PROTEAN TGX Stain-Free (Bio-Rad). The purest fractions were pooled, concentrated together, flash frozen and stored at $-80^{\circ} \mathrm{C}$.

Drug panel and other reagents. The library of FDA-approved drugs was from Selleck (L1300). For further testing, single drugs were purchased as listed in Supplemental Table 4. Thienopyridone was generously provided by Dr. Zhong-Yin Zhang (Purdue University). PRL-3 overexpressing construct was made by cloning full length PRL-3 cDNA into p3XFLAG-CMV-14 expression vector (Sigma, Cat \# E7908).

In vitro phosphatase assay. In 384 well plates, $2.5 \mu \mathrm{M}$ recombinant PRL-1, PRL-2, or PRL-3 was combined with DiFUMP (Life Technologies, Cat \# E12020) at the $\mathrm{K}_{\mathrm{M}}$ of each protein in reaction buffer $(20 \mathrm{mM}$ Tris-Cl, pH 7.5, $150 \mathrm{mM} \mathrm{NaCl}, 10 \mathrm{mM}$ DTT) as previously reported ${ }^{41}$. Briefly, protein was diluted in reaction buffer and allowed to incubate at $4{ }^{\circ} \mathrm{C}$ for $20 \mathrm{~min}$ to allow for full reduction of the active site. DiFMUP, drug, and protein were added, and plates were incubated for $20 \mathrm{~min}$ at room temperature. Fluorescence intensities were measured on a Cytation 5 plate reader (Biotek) at an EX:360 nm and EM:460 nm. Data were normalized to vehicle control and $\mathrm{IC}_{50}$ values were calculated on GraphPad Prism version 8. Inhibition selectivity for PRLs over a panel of other phosphatases was determined using PhosphataseProfiler (Eurofins, Cat \# PP260).

Cell culture. HEK293T, HCT116, and SW480 human cell lines, from ATCC, (CRL-3216, CCL-247, and CCL-228 respectively) were maintained in 1X DMEM with glutamine and glucose (Gibco, Cat \# 11,965-092) supplemented with 10\% FBS (Atlanta Biologicals, Cat \# S11150H) and Penicillin/Streptomycin (Fisher Scientific Cat $\#, 10,378,016)$. Cells were cultured at $37^{\circ} \mathrm{C}$ in a humidified incubator with $5 \% \mathrm{CO}_{2}$.

PRL-3 overexpression and shRNA knockdown. pENTR-PRL-3 (previously described ${ }^{51}$ ) was cloned into pLKO-CMV-puro using LR clonase (Thermofisher, 11,791,019) according to manufacturer's directions. pLKO-CMV empty vector (EV) and pLKO-CMV:PRL-3 were transfected into HEK293T using Lipofectamine 3000 (ThermoFisher, L3000015) according to manufacturer's protocol. Transfected cells were selected with $1 \mu \mathrm{g} /$ $\mathrm{mL}$ puromycin in complete media to establish the cell line, then were maintained under $0.5 \mu \mathrm{g} / \mathrm{mL}$ puromycin in complete media. For PRL-3 knockdown, pLKO.1-scrambled shRNA and pLKO.1-PRL-3 shRNA ${ }^{51}$ plasmids were transfected into HCT116 cells using Lipofectamine 3000 (Thermo Fisher, Cat \# L3000015) according to the manufacturer's directions. Cells were selected for $48 \mathrm{~h}$ in $1 \mu \mathrm{g} / \mathrm{mL}$ puromycin before use in assays.

Scratch assay. Cells were seeded into duplicate wells of a 48 well plates at a density of $2 \times 10^{5}$ cells per well for HEK293T or $4 \times 10^{5}$ for HCT116 and SW480. Cells were recovered overnight and were scratched using a p20 pipette tip the following day. Compounds were added at the concentrations indicated. Images were obtained immediately after the scratch and after a $24 \mathrm{~h}$ incubation with the drug on an Evos FL inverted microscope (ThermoFisher). The area within the scratch was calculated using ImageJ. The area migrated was measured as the difference in wound area at $0 \mathrm{~h}$ and $24 \mathrm{~h}$ and the percent change in migration was calculated by dividing the difference in wound area of treatment by the difference in wound area of the control.

Viability assay. Cells were seeded into duplicate wells of a 96 well plate at a density of $2 \times 10^{4}$ cells per well and allowed to recover for $24 \mathrm{~h}$. Cells were then treated with drug or DMSO at the indicated concentrations for $16 \mathrm{~h}$. Following drug treatment, $10 \mu \mathrm{L}$ of $5 \mathrm{mg} / \mathrm{ml}$ MTT (Sigma, M5655) was added to each well and cells were 
incubated for $4 \mathrm{~h}$. Finally, media were removed, and the dye was solubilized in $100 \mu \mathrm{L}$ of $0.1 \mathrm{M} \mathrm{HCl}$ in isopropanol. Absorbance was measured at $570 \mathrm{~nm}$ and $690 \mathrm{~nm}$ with final absorbance measurements as $570-690 \mathrm{~nm}$.

Click-IT Edu assay. The cell proliferation assay was performed using the Click-IT Edu cell proliferation kit from (Thermo Fisher, Cat \# C10419) according to the manufacturer's protocol. Briefly, HEK293T, HCT116 and SW480 cells were plated in 6 well dishes at $4 \times 10^{5}$ cell seeding density and treated with DMSO, Candesartan and Salirasib at the indicated concentrations. Edu was added $2 \mathrm{~h}$ prior to collecting the cells at $24 \mathrm{~h}$ and $48 \mathrm{~h}$ time points and the assay was performed according to the instructions from the manual. Flow cytometry with AlexaFluor647 conjugated azide dye was used to determine the cellular proliferation and DAPI was used for the DNA content.

Annexin V staining. HEK293T, HCT116, and SW480 cells were plated in 6 well dishes at $4 \times 10^{5}$ cell seeding density and treated with DMSO, Candesartan and Salirasib at the indicated concentrations. Cells were collected at $24 \mathrm{~h}$ and $48 \mathrm{~h}$ time points and the AnnexinV staining was performed according to the instructions from the manual (Thermo Fisher, Cat \# 00-0055-56). Flow cytometry with AnnexinV-FITC conjugated antibody (Thermo Fisher, Cat \# A13199) was used to determine the population of cells incorporating Annexin V and PI was used for the DNA content.

Western Blot. Cells were lysed using Qproteome lysis buffer (Qiagen, Cat \# 37901), then spun at 14,000 rpm for $10 \mathrm{~min}$ at $4{ }^{\circ} \mathrm{C}$. Protein concentration in the supernatant was quantified using BCA assay (Thermo Scientific, Cat \# 23227). $30 \mu \mathrm{g}$ of protein was loaded into each lane of a TGX-stain-free pre-cast 4-20\% SDS gel (Biorad, Cat \# 4568094), total protein was quantified upon stain-free gel imaging, and protein was transferred onto PVDF membrane using the Trans-Blot Turbo Transfer System (BioRad, Cat \# 1704150). Membranes were blocked with $5 \%$ milk in 1\% TBST for $1 \mathrm{~h}$, and a 1:1000 dilution of Anti-PRL-3 antibody (R\&D Biosystems, Cat \# MAB3219), 1:5000 phospho-ERK (Cell Signaling, Cat \# 19101) or 1:5000 total ERK (Cell Signaling, Cat \# 137F5) was added overnight. Following three washes in TBST, secondary HRP-conjugated antibody (GE, Cat \# NA9340V) were added at a 1:5000 dilution for $1 \mathrm{~h}$ and membranes imaged using Clarity Western ECL Substrate. (BioRad, Cat \# 1705061).

Molecular docking. The molecular docking software, Autodock41-3 (version 4.2.6) was used to identify probable binding sites. Protein structures were obtained from published NMR solution structures of apo (PDB code: 1V3A4) and vanadate-bound (PDB code: 2MBC5, model 1) PRL-3. Ligand sdf files (pubchem.com6) were converted to PDB using OpenBabel7.8. Ligand and receptor files were prepared and Gasteiger charges added using AutodockTools1. Blind Autodock docking was performed by covering the entire protein structure with a grid box consisting of $126 \times 126 \times 126$ points and centered at the center of the macromolecule. Docking was then performed without bias to search for all possible binding sites using Lamarckian Genetic Algorithm with a rigid protein and flexible ligands. Population size was set to 300 and the maximum number of energy evaluations and maximum number of generations were set to 30,000,000 and 27,000, respectively. For each ligand, 100 dockings were performed and clustered using an RMSD tolerance of $2 \AA$. In addition to the set of FDA-approved drugs, dexamethasone and PRL inhibitor I, which were used as negative and positive controls in experimental work, respectively, as well as a farnesyl tail were docked following the same protocols. Visualizations were performed using AutodockTools and PyMol10.

Statistics. All experiments, expect for the drug screen, were performed in biological triplicate across at least three independent time points with at least two technical replicates. Where applicable, experimental values were normalized to vehicle control, \pm standard deviation. Statistical tests were run in Graphpad Prism Version 9. $p$ values were calculated either using one-way ANOVA with Tukey HSD or two-way ANOVA with Dunnett test for multiple comparisons, as noted in the figure legends. Changes were considered significant if $p<0.05$.

\section{Data availability}

All data generated or analyzed during this study are included in this article and its supplementary files. The datasets that were analyzed are available from the corresponding authors on reasonable request.

Received: 15 May 2020; Accepted: 29 April 2021

Published online: 13 May 2021

\section{References}

1. Ruckert, M. T., de Andrade, P. V., Santos, V. S. \& Silveira, V. S. Protein tyrosine phosphatases: promising targets in pancreatic ductal adenocarcinoma. Cell Mol. Life Sci. 76, 2571-2592. https://doi.org/10.1007/s00018-019-03095-4 (2019).

2. Aceto, N. et al. Tyrosine phosphatase SHP2 promotes breast cancer progression and maintains tumor-initiating cells via activation of key transcription factors and a positive feedback signaling loop. Nat. Med. 18, 529-537. https://doi.org/10.1038/nm.2645 (2012).

3. Hoekstra, E. et al. Increased PTP1B expression and phosphatase activity in colorectal cancer results in a more invasive phenotype and worse patient outcome. Oncotarget 7, 21922-21938. https://doi.org/10.18632/oncotarget.7829 (2016).

4. Hu, Z., Li, J., Gao, Q., Wei, S. \& Yang, B. SHP2 overexpression enhances the invasion and metastasis of ovarian cancer in vitro and in vivo. Oncol. Targets Ther. 10, 3881-3891. https://doi.org/10.2147/ott.s138833 (2017).

5. Lessard, L. et al. PTP1B is an androgen receptor-regulated phosphatase that promotes the progression of prostate cancer. Cancer Res. 72, 1529-1537. https://doi.org/10.1158/0008-5472.can-11-2602 (2012).

6. Bessette, D. C., Qiu, D. \& Pallen, C. J. PRL PTPs: mediators and markers of cancer progression. Cancer Metastasis Rev. 27, $231-252$. https://doi.org/10.1007/s10555-008-9121-3 (2008). 
7. Bardelli, A. et al. PRL-3 expression in metastatic cancers. Clin. Cancer Res. 9, 5607-5615 (2003).

8. Campbell, A. M. \& Zhang, Z. Y. Phosphatase of regenerating liver: a novel target for cancer therapy. Expert Opin. Ther. Targets 18, 555-569. https://doi.org/10.1517/14728222.2014.892926 (2014).

9. Dai, N., Lu, A. P., Shou, C. C. \& Li, J. Y. Expression of phosphatase regenerating liver 3 is an independent prognostic indicator for gastric cancer. World J. Gastroenterol. 15, 1499-1505. https://doi.org/10.3748/wjg.15.1499 (2009).

10. den Hollander, P. et al. Phosphatase PTP4A3 promotes triple-negative breast cancer growth and predicts poor patient survival. Cancer Res. 76, 1942-1953. https://doi.org/10.1158/0008-5472.can-14-0673 (2016).

11. Park, J. E. et al. Oncogenic roles of PRL-3 in FLT3-ITD induced acute myeloid leukaemia. EMBO Mol. Med. 5, 1351-1366. https:// doi.org/10.1002/emmm.201202183 (2013).

12. Saha, S. et al. A phosphatase associated with metastasis of colorectal cancer. Science 294, 1343-1346. https://doi.org/10.1126/scien ce.1065817 (2001).

13. Vandsemb, E. N. et al. Phosphatase of regenerating liver 3 (PRL-3) is overexpressed in human prostate cancer tissue and promotes growth and migration. J. Transl. Med. 14, 71. https://doi.org/10.1186/s12967-016-0830-z (2016).

14. Wang, L. et al. PTP4A3 is a target for inhibition of cell proliferatin, migration and invasion through Akt/mTOR signaling pathway in glioblastoma under the regulation of miR-137. Brain Res. 1646, 441-450. https://doi.org/10.1016/j.brainres.2016.06.026 (2016).

15. Yeh, H. C. et al. PTP4A3 independently predicts metastasis and survival in upper tract urothelial carcinoma treated with radical nephroureterectomy. J. Urol. 194, 1449-1455. https://doi.org/10.1016/j.juro.2015.05.101 (2015).

16. Thura, M. et al. PRL3-zumab as an immunotherapy to inhibit tumors expressing PRL3 oncoprotein. Nat. Commun. 10, 2484. https://doi.org/10.1038/s41467-019-10127-x (2019).

17. Beekman, R. et al. Retroviral integration mutagenesis in mice and comparative analysis in human AML identify reduced PTP4A3 expression as a prognostic indicator. PLoS ONE 6, e26537. https://doi.org/10.1371/journal.pone.0026537 (2011).

18. Mayinuer, A. et al. Upregulation of protein tyrosine phosphatase type IVA member 3 (PTP4A3/PRL-3) is associated with tumor differentiation and a poor prognosis in human hepatocellular carcinoma. Ann. Surg. Oncol. 20, 305-317. https://doi.org/10.1245/ s10434-012-2395-2 (2013).

19. Qu, S. et al. Independent oncogenic and therapeutic significance of phosphatase PRL-3 in FLT3-ITD-negative acute myeloid leukemia. Cancer 120, 2130-2141. https://doi.org/10.1002/cncr.28668 (2014).

20. Ren, T. et al. Prognostic significance of phosphatase of regenerating liver-3 expression in ovarian cancer. Pathol. Oncol. Res. 15, 555-560. https://doi.org/10.1007/s12253-009-9153-1 (2009).

21. Guo, K. et al. Catalytic domain of PRL-3 plays an essential role in tumor metastasis: formation of PRL-3 tumors inside the blood vessels. Cancer Biol. Ther. 3, 945-951. https://doi.org/10.4161/cbt.3.10.1111 (2004).

22. Kato, H. et al. High expression of PRL-3 promotes cancer cell motility and liver metastasis in human colorectal cancer: a predictive molecular marker of metachronous liver and lung metastases. Clin. Cancer Res. 10, 7318-7328. https://doi.org/10.1158/1078-0432. ccr-04-0485 (2004).

23. Wu, X. et al. Phosphatase of regenerating liver-3 promotes motility and metastasis of mouse melanoma cells. Am. J. Pathol. 164, 2039-2054. https://doi.org/10.1016/s0002-9440(10)63763-7 (2004).

24. Zeng, Q. et al. PRL-3 and PRL-1 promote cell migration, invasion, and metastasis. Cancer Res. 63, 2716-2722 (2003).

25. Li, Z. et al. Inhibition of PRL-3 gene expression in gastric cancer cell line SGC7901 via microRNA suppressed reduces peritoneal metastasis. Biochem. Biophys. Res. Commun. 348, 229-237. https://doi.org/10.1016/j.bbrc.2006.07.043 (2006).

26. Polato, F. et al. PRL-3 phosphatase is implicated in ovarian cancer growth. Clin. Cancer Res. 11, 6835-6839. https://doi.org/10. 1158/1078-0432.ccr-04-2357 (2005).

27. Qian, F. et al. PRL-3 siRNA inhibits the metastasis of B16-BL6 mouse melanoma cells in vitro and in vivo. Mol. Med. 13, 151-159. https://doi.org/10.2119/2006-00076.Qian (2007).

28. Zimmerman, M. W., Homanics, G. E. \& Lazo, J. S. Targeted deletion of the metastasis-associated phosphatase Ptp4a3 (PRL-3) suppresses murine colon cancer. PLoS ONE 8, e58300. https://doi.org/10.1371/journal.pone.0058300 (2013).

29. Hardy, S. et al. The protein tyrosine phosphatase PRL-2 interacts with the magnesium transporter CNNM3 to promote oncogenesis. Oncogene 34, 986-995. https://doi.org/10.1038/onc.2014.33 (2015).

30. Jin, S. et al. Oncogenic function and prognostic significance of protein tyrosine phosphatase PRL-1 in hepatocellular carcinoma. Oncotarget 5, 3685-3696. https://doi.org/10.18632/oncotarget.1986 (2014).

31. Liu, L. Z. et al. Protein tyrosine phosphatase PTP4A1 promotes proliferation and epithelial-mesenchymal transition in intrahepatic cholangiocarcinoma via the PI3K/AKT pathway. Oncotarget 7, 75210-75220. https://doi.org/10.18632/oncotarget.12116 (2016).

32. Shinmei, S. et al. Identification of PRL1 as a novel diagnostic and therapeutic target for castration-resistant prostate cancer by the Escherichia coli ampicillin secretion trap (CAST) method. Urol. Oncol. 32, 769-778. https://doi.org/10.1016/j.urolonc.2014.03. 007 (2014).

33. Wang, Y. \& Lazo, J. S. Metastasis-associated phosphatase PRL-2 regulates tumor cell migration and invasion. Oncogene 31, 818-827. https://doi.org/10.1038/onc.2011.281 (2012).

34. Dong, J., Sui, L., Wang, Q., Chen, M. \& Sun, H. MicroRNA-26a inhibits cell proliferation and invasion of cervical cancer cells by targeting protein tyrosine phosphatase type IVA 1. Mol. Med. Rep. 10, 1426-1432. https://doi.org/10.3892/mmr.2014.2335 (2014).

35. Dumaual, C. M. et al. Tissue-specific alterations of PRL-1 and PRL-2 expression in cancer. Am. J. Transl. Res. 4, 83-101 (2012).

36. Hardy, S., Wong, N. N., Muller, W. J., Park, M. \& Tremblay, M. L. Overexpression of the protein tyrosine phosphatase PRL-2 correlates with breast tumor formation and progression. Cancer Res. 70, 8959-8967. https://doi.org/10.1158/0008-5472.can-10-2041 (2010).

37. Rios, P., Li, X. \& Kohn, M. Molecular mechanisms of the PRL phosphatases. Febs J. 280, 505-524. https://doi.org/10.1111/j.17424658.2012.08565.x (2013).

38. Wei, M., Korotkov, K. V. \& Blackburn, J. S. Targeting phosphatases of regenerating liver (PRLs) in cancer. Pharmacol. Ther. 190, 128-138. https://doi.org/10.1016/j.pharmthera.2018.05.014 (2018).

39. Bai, Y. et al. Novel anticancer agents based on targeting the trimer interface of the PRL phosphatase. Cancer Res. 76, 4805-4815. https://doi.org/10.1158/0008-5472.can-15-2323 (2016).

40. Daouti, S. et al. A selective phosphatase of regenerating liver phosphatase inhibitor suppresses tumor cell anchorage-independent growth by a novel mechanism involving p130Cas cleavage. Cancer Res. 68, 1162-1169. https://doi.org/10.1158/0008-5472.can07-2349 (2008).

41. Hoeger, B., Diether, M., Ballester, P. J. \& Kohn, M. Biochemical evaluation of virtual screening methods reveals a cell-active inhibitor of the cancer-promoting phosphatases of regenerating liver. Eur. J. Med. Chem. 88, 89-100. https://doi.org/10.1016/j.ejmech. 2014.08.060 (2014).

42. McQueeney, K. E. et al. Targeting ovarian cancer and endothelium with an allosteric PTP4A3 phosphatase inhibitor. Oncotarget 9, 8223-8240. https://doi.org/10.18632/oncotarget.23787 (2018).

43. Salamoun, J. M. \& Wipf, P. Allosteric modulation of phosphatase activity may redefine therapeutic value. J. Med. Chem. 59, 7771-7772. https://doi.org/10.1021/acs.jmedchem.6b01210 (2016).

44. Thura, M. et al. PRL3-zumab, a first-in-class humanized antibody for cancer therapy. JCI Insight 1, e87607. https://doi.org/10. $1172 /$ jci.insight. 87607 (2016).

45. Ahn, J. H. et al. Synthesis and biological evaluation of rhodanine derivatives as PRL-3 inhibitors. Bioorg. Med. Chem. Lett. 16, 2996-2999. https://doi.org/10.1016/j.bmcl.2006.02.060 (2006). 
46. Souers, A. J. et al. ABT-199, a potent and selective BCL-2 inhibitor, achieves antitumor activity while sparing platelets. Nat. Med. 19, 202-208. https://doi.org/10.1038/nm.3048 (2013).

47. Ding, Q. et al. Discovery of RG7388, a potent and selective p53-MDM2 inhibitor in clinical development. J. Med. Chem. 56, 5979-5983. https://doi.org/10.1021/jm400487c (2013).

48. Nakahara, T. et al. YM155, a novel small-molecule survivin suppressant, induces regression of established human hormonerefractory prostate tumor xenografts. Cancer Res. 67, 8014-8021. https://doi.org/10.1158/0008-5472.can-07-1343 (2007).

49. Ming, J., Liu, N., Gu, Y., Qiu, X. \& Wang, E. H. PRL-3 facilitates angiogenesis and metastasis by increasing ERK phosphorylation and up-regulating the levels and activities of Rho-A/C in lung cancer. Pathology 41, 118-126. https://doi.org/10.1080/0031302080 2579268 (2009).

50. Mu, N. et al. PRL-3 is a potential glioblastoma prognostic marker and promotes glioblastoma progression by enhancing MMP7 through the ERK and JNK pathways. Theranostics 8, 1527-1539. https://doi.org/10.7150/thno.22699 (2018).

51. Wei, M., Haney, M. G., Rivas, D. R. \& Blackburn, J. S. Protein tyrosine phosphatase 4A3 (PTP4A3/PRL-3) drives migration and progression of T-cell acute lymphoblastic leukemia in vitro and in vivo. Oncogenesis $\mathbf{9}, 6$. https://doi.org/10.1038/s41389-0200192-5 (2020).

52. Hjort, M. A. et al. Phosphatase of regenerating liver-3 is expressed in acute lymphoblastic leukemia and mediates leukemic cell adhesion, migration and drug resistance. Oncotarget 9, 3549-3561. https://doi.org/10.18632/oncotarget.23186 (2018).

53. Zhou, J. et al. The pro-metastasis tyrosine phosphatase, PRL-3 (PTP4A3), is a novel mediator of oncogenic function of BCR-ABL in human chronic myeloid leukemia. Mol. Cancer 11, 72. https://doi.org/10.1186/1476-4598-11-72 (2012).

54. Koveal, D., Clarkson, M. W., Wood, T. K., Page, R. \& Peti, W. Ligand binding reduces conformational flexibility in the active site of tyrosine phosphatase related to biofilm formation A (TpbA) from Pseudomonasaeruginosa. J. Mol. Biol. 425, 2219-2231. https:// doi.org/10.1016/j.jmb.2013.03.023 (2013).

55. Whittier, S. K., Hengge, A. C. \& Loria, J. P. Conformational motions regulate phosphoryl transfer in related protein tyrosine phosphatases. Science 341, 899-903. https://doi.org/10.1126/science.1241735 (2013).

\section{Acknowledgements}

We thank Dr. Vivek Ragnakar (University of Kentucky) for providing the FDA approved library, and ZhongYing Zhang (Purdue University) for providing Thienopryridone. We thank Konstantin Korotkov (University of Kentucky) for assistance with generating Pymol structures. This research was supported by a St. Baldrick's Foundation Research Grant, and NIH grants DP2CA228043 and R37CA227656 (to J.S. Blackburn). The research was also supported by the James Graham Brown Cancer Center (to D. Lee).

\section{Author contributions}

D.R.R. and J.S.B. conceived of and designed the study. D.R.R. performed all biochemical experiments with B.P. assisting on some assays. C.N.S. generated all proteins used in this study. S.S. carried out all cell-based assays. M.D.C and D.L. designed and carried out molecular docking analyses and wrote the corresponding portion of the manuscript. D.R.R., M.D.C., and S.S. drafted the manuscript, J.S.B and D.L. revised. All authors have read, edited, and approved of the final version of this manuscript.

\section{Competing interests}

The authors declare no competing interests.

\section{Additional information}

Supplementary Information The online version contains supplementary material available at https://doi.org/ 10.1038/s41598-021-89668-5.

Correspondence and requests for materials should be addressed to D.L. or J.S.B.

Reprints and permissions information is available at www.nature.com/reprints.

Publisher's note Springer Nature remains neutral with regard to jurisdictional claims in published maps and institutional affiliations.

Open Access This article is licensed under a Creative Commons Attribution 4.0 International License, which permits use, sharing, adaptation, distribution and reproduction in any medium or format, as long as you give appropriate credit to the original author(s) and the source, provide a link to the Creative Commons licence, and indicate if changes were made. The images or other third party material in this article are included in the article's Creative Commons licence, unless indicated otherwise in a credit line to the material. If material is not included in the article's Creative Commons licence and your intended use is not permitted by statutory regulation or exceeds the permitted use, you will need to obtain permission directly from the copyright holder. To view a copy of this licence, visit http://creativecommons.org/licenses/by/4.0/.

(C) The Author(s) 2021 